\title{
ON THE SOLVABILITY OF NONLINEAR NONCOMPACT OPERATOR EQUATIONS
}

\author{
E. U. TARAFDAR and H. B. THOMPSON
}

(Received 4 February 1986)

Communicated by R. O. Vyborny

\begin{abstract}
The notion of $(p, k)$-epi mappings is introduced. The properties of such mappings are studied and the results obtained are applied to some differential equations.

1980 Mathematics subject classification (Amer. Math. Soc.): primary $47 \mathrm{H} 15$; secondary $47 \mathrm{H} \mathrm{10,34}$ K 10, 35 L 15.

Keywords and phrases: admissible mapping, $p$-epi, $(p, k)$-epi, $k$-set contraction, measure of unsolvability.
\end{abstract}

\section{Introduction}

Furi, Martelli and Vignoli [1] have introduced the notion of $p$-epi mappings as follows:

If $E$ and $F$ are normed linear spaces, $\Omega \subset E$ is a bounded open set and $p \in F$ then a continuous mapping $f: \bar{\Omega} \rightarrow F$ with $f(x) \neq p$ for any $x \in \partial \Omega$ is called $p$-epi if for each compact mapping $h: \bar{\Omega} \rightarrow F$ with $h \equiv 0$ on $\partial \Omega$, the equation $f(x)=h(x)+p$ has a solution in $\Omega$.

In their paper the authors have shown that the p-epi mappings have 'existence', 'boundary dependence', 'normalization', 'localization' and 'homotopy' properties similar to those of topological degree theory. The theory of $p$-epi mappings is based on elementary tools such as the Schauder fixed point theorem, Urysohn's lemma, etc.

(c) Copyright Australian Mathematical Society 1987, Serial-fee code 0334-2700/87 
Unlike the case of degree theory $p$-epi mappings may act between different spaces. These theories are normally used to establish the existence of solutions of nonlinear problems. However in applications such as to differential and functional differential equations the problems occur frequently as nonlinear mappings acting between different spaces. Thus the theory of $p$-epi mappings is directly applicable to such problems while to apply degree theory it is necessary to reformulate the problems as nonlinear self mappings acting on some space.

In this paper we have introduced the concept of a $(p, k)$-epi mapping by allowing the mapping $h$ to be a $k$-set contraction rather than just a compact mapping and requiring $E$ and $F$ to be Banach spaces. Thus we are dealing with a class of mappings smaller than that of $p$-epi mappings. However, by restricting $f$ to be a $(p, k)$-epi mapping we can solve the equation $f(x)=p+h(x)$ for more general mappings $h$; this is necessary in some applications.

We have applied our theory of $(p, k)$-epi mappings to a number of problems.

The general outline of our paper is as follows:

Section 1-preliminaries and background work;

Section $2-(p, k)$-epi mappings and their properties;

Section 3- $(p, k)$-epi mappings on the whole space;

Section 4-applications.

\section{Preliminaries. Measure of noncompactness and set contraction}

In this section we mention the well-known concepts of measure of noncompactness and $k$-set contractions and some of their properties. We also prove some results which we will need for the subsequent development of our theory. Although the section may be considered as ground work, some results however have their own independent interest. We should point out that some of the results in this section are not new. However, we include their proofs for the completeness of presentation and convenience of the readers.

Definition 1.1. Let $X$ be a metric space and $A \subset X$ a bounded subset. Set $\Delta(A)=\{\varepsilon>0: A$ can be covered by a finite number of sets of diameter less than $\varepsilon\}$. Then $\alpha(A)=\inf \Delta(A)$ is defined to be the measure of noncompactness of $A$. This notion of measure of noncompactness was introduced by Kuratowski [6].

Another notion of measure of noncompactness was introduced by Gohberg, Goldstein and Markus [3] in the following manner:

Let $\sigma(A)=\{\varepsilon>0: A$ can be covered by a finite number of balls of radius $\varepsilon$. Then $\beta(A)=\inf \sigma(A)$ is called the (ball) measure of noncompactness of $A$. 
In what follows we deal only with the measure of noncompactness $\alpha$. However, everything we do here will also hold with the measure of noncompactness $\beta$ and, for that matter, with any other measure of noncompactness so long as it has the basic properties of a measure of noncompactness.

Properties of the measure of noncompactness. Let $A$ and $B$ be bounded subsets of a metric space $X$. Then

(1) $\alpha(A)=0$ if and only if $A$ is relatively compact;

(2) $A \subseteq B$ implies $\alpha(A) \leqslant \alpha(B)$;

(3) $\alpha(A)=\alpha(\bar{A})$, where $\bar{A}$ denotes the closure of $A$;

(4) $\alpha(A \cup B)=\max (\alpha(A), \alpha(B))$.

Furthermore, if $X$ is a normed space, then

(5) $\alpha(\operatorname{Co} A)=\alpha(A)$ where $\operatorname{Co} A$ denotes the convex hull of $A$, and

(6) $\alpha(A+B) \leqslant \alpha(A)+\alpha(B)$.

For proof of these we refer to Lloyd [7] or Martin [8].

Definition 1.2. A continuous mapping $f: X \rightarrow Y$ of a metric space $X$ into a metric space $Y$ is said to be a $k$-set contraction if for each bounded subset $A$ of $X$, $\alpha(f(A)) \leqslant k \alpha(A)$, where $k \geqslant 0$, and is said to be a condensing mapping if for each noncompact bounded subset $A$ of $X, \alpha(f(A))<\alpha(A)$.

In the sequel we denote by $\delta(A)$ the diameter of a bounded subset $A$ of a metric space $X$.

LEMMA 1.1. Let $\Omega$ be a nonempty bounded subset of a metric space $X$ and let $A \subseteq[0,1] \times \Omega$. Let $\pi(A)=\{x \in \Omega:(t, x) \in A$ for some $t \in[0,1]\}$. Then $\alpha(\pi(A))=\alpha(A)$.

Proof. We first show that $\alpha(\pi(A)) \leqslant \alpha(A)$. Let $\varepsilon>0$. Then there exists a finite number of subsets $D_{1}, D_{2}, \ldots, D_{n}$ of $A$ with $\delta\left(D_{i}\right) \leqslant \alpha(A)+\varepsilon$ for $i=$ $1,2, \ldots, n$ such that $A \subseteq \bigcup_{i=1}^{n} D_{i}$. Clearly $\pi(A) \subseteq \bigcup_{i=1}^{n} \pi\left(D_{i}\right)$ and $\delta\left(\pi\left(D_{i}\right)\right) \leqslant$ $\delta\left(D_{i}\right) \leqslant \alpha(A)+\varepsilon$. Thus $\alpha(\pi(A)) \leqslant \alpha(A)+\varepsilon$. Since $\varepsilon>0$ is arbitrary, $\alpha(\pi(A))$ $\leqslant \alpha(A)$. Now $A \subseteq[0,1] \times \pi(A)$ so $\alpha(A) \leqslant \alpha([0,1] \times \pi(A))$. Thus it suffices to show that $\alpha([0,1] \times \pi(A)) \leqslant \alpha(A)$. Now given $\varepsilon>0$ there exist a finite number of subsets $D_{i}$ of $\pi(A)$ with $\delta\left(D_{i}\right) \leqslant \alpha(\pi(A))+\varepsilon / 2$ for $i=1, \ldots, m$ such that $\pi(A) \subseteq \bigcup_{i-1}^{m} D_{i}$. Let $T_{i}=[(i-1) / l, i / l]$ for $i=1, \ldots, l$ where $1 / l<\varepsilon / 2$. Thus $[0,1] \times \pi(A) \subseteq \bigcup_{1 \leqslant i \leqslant m, 1 \leqslant j \leqslant l} T_{j} \times D_{i}$ and $\delta\left(T_{j} \times D_{i}\right) \leqslant \alpha(\pi(A))+\varepsilon$. Thus $\alpha([0,1] \times \pi(A)) \leqslant \alpha(\pi(A))+\varepsilon$. Since $\varepsilon$ was arbitrary, the result follows.

TheOrem 1.1. Let $X$ and $Y$ be metric spaces and $\Omega$ a bounded subset of $X$. Let $H:[0,1] \times \Omega \rightarrow Y$ be a $k$-set contraction and $\phi: \Omega \rightarrow[0,1]$ be a continuous mapping. The mapping $h: \Omega \rightarrow Y$ defined by $h(x)=H(\phi(x), x), x \in \Omega$, is a $k$-set 
contraction. (Throughout this paper we use the metric $\rho(\cdot, \cdot)$ on $[0,1] \times X$ defined by $\rho((t, x),(\bar{t}, \bar{x}))=\max (|t-\bar{t}|, d(x, \bar{x}))$ where $d$ is the metric on $X$.)

PROOF. Let $S \subset \Omega$, then $\alpha(h(S)) \leqslant \alpha(H(\phi(S) \times S)) \leqslant k \alpha(\phi(S) \times S)=$ $k \alpha(S)$ by Lemma 1.1. As $h$ is continuous, the proof is complete.

We need the following two lemmas to prove our next theorem.

LEMMA 1.2. Let $A$ be a nonempty bounded subset of a metric space $(X, \rho)$ and for $\varepsilon>0$, let $B(A, \varepsilon)=\bigcup_{x \in A} B(x, \varepsilon)$ where $B(x, \varepsilon)=\{y \in A: \rho(x, y)<\varepsilon\}$. Then $\delta(B(A, \varepsilon)) \leqslant \delta(A)+2 \varepsilon$. As before, $\delta$ stands for diameter.

Proof. Let $u, v \in B(A, \varepsilon)$. Then $u \in B(x, \varepsilon)$ and $v \in B(y, \varepsilon)$ for some $x$, $y \in A$. Hence $\rho(u, v) \leqslant \rho(u, x)+\rho(x, y)+\rho(y, v)<\delta(A)+2 \varepsilon$.

Lemma 1.3. Let $A$ be as in Lemma 1.2. Then $\alpha(B(A, \varepsilon)) \leqslant \alpha(A)+2 \varepsilon$.

Proof. This is immediate from Lemma 1.2 and definition of $\alpha$.

For the rest of the paper, unless otherwise stated $E$ and $F$ will denote real Banach spaces and $\Omega$ an open bounded subset of $E$. We will also denote by $\Sigma_{k}(\bar{\Omega})$ the set of all $k$-set contractions $f: \bar{\Omega} \rightarrow F$.

THEOREM 1.2. Let $h:[0,1] \rightarrow \Sigma_{k}(\bar{\Omega})$ be a continuous mapping where $\Sigma_{k}(\bar{\Omega})$ is equipped with the topology of uniform convergence. Let $H:[0,1] \times \bar{\Omega} \rightarrow F$ be defined by $H(t, x)=h(t)(x)$. Then $H$ is a $k$-set contraction.

Proof. Let $S \subseteq[0,1] \times \bar{\Omega}$. Then clearly $H(S) \subseteq H([0,1] \times \pi(S))$. Given $\varepsilon>0$, there exists, by uniform continuity of $h$, points $t_{1}, t_{2}, \ldots, t_{m+1}$ with $0=t_{1}<t_{2}$ $<\cdots<t_{m}<t_{m+1}=1$ such that $\|h(t)(x)-h(\tau)(x)\|<\varepsilon$ whenever $t, \tau \in\left[t_{i}\right.$, $\left.t_{i+1}\right], 1 \leqslant i \leqslant m, x \in \bar{\Omega}$. Thus for $(t, x) \in[0,1] \times \pi(S)$, there is $t_{i}, 1 \leqslant i \leqslant m$ such that $\left\|h\left(t_{i}\right)(x)-h(t)(x)\right\|<\varepsilon$. Hence it follows that

$$
H([0,1] \times \pi(S)) \subseteq \bigcup_{i=1}^{m} B\left(h\left(t_{i}\right)(\pi(S)), \varepsilon\right)
$$

However

$$
\begin{aligned}
\alpha\left(h\left(t_{i}\right)(\pi(S))\right) & \leqslant k \alpha(\pi(S)) \text { for } i=1,2, \ldots, m \\
& \leqslant k \alpha(S) \text { by Lemma } 1.1 .
\end{aligned}
$$


Hence using (1), Lemma 1.3 and properties of measure of noncompactness we obtain $\alpha(H(S)) \leqslant \alpha(H([0,1] \times \pi(S))) \leqslant k \alpha(S)+2 \varepsilon$. But since $\varepsilon>0$ is arbitrary, the theorem is proved.

THEOREM 1.3. Let $E$ and $F$ be normed spaces and $\Omega \subset E$ a bounded open set. Let $h: \bar{\Omega} \rightarrow F$ be a $k$-set contraction such that $h(x)=0$ for all $x \in \partial \Omega$, where $\partial \Omega$ denotes the boundary of $\Omega$. Let $\bar{h}: E \rightarrow F$ be defined by

Then $\bar{h}$ is a $k$-set contraction.

$$
\bar{h}(x)= \begin{cases}h(x) & \text { for } x \in \bar{\Omega}, \\ 0 & \text { for } x \notin \bar{\Omega} .\end{cases}
$$

Proof. Let $S \subset E$ be a bounded set. If $S \cap \Omega=\varnothing$ then $\bar{h}(S)=0$. So $\alpha(\bar{h}(S))=0 \leqslant k \alpha(S)$. Now let $S \cap \Omega \neq \varnothing$. Then $\alpha(\bar{h}(S \cap \Omega))=\alpha(h(S \cap \Omega))$ $\leqslant k \alpha(S \cap \Omega) \leqslant k \alpha(S)$ (by property 2). Also $\bar{h}(S) \subseteq \bar{h}(S \cap \Omega) \cup\{0\}$. Hence $\alpha(\bar{h}(S)) \leqslant \alpha(\bar{h}(S \cap \Omega)) \leqslant k \alpha(S)$. Thus the result follows as $\bar{h}$ is continuous.

Theorem 1.4. (1) (Fixed Point Theorem of Darbo). If $D \subset E$ is a closed bounded convex set and $f: D \rightarrow D$ is a $k$-set contraction with $k \in[0,1)$, then $f$ has a fixed point.

(2) If $D$ is as above and $f: D \rightarrow D$ is a condensing mapping, then $f$ has a fixed point.

Proof. See Martin [8], pp. 125-127.

We will also need the following notion of a $k$-proper mapping. We first recall that a continuous mapping $f: X \rightarrow Y$ of a topological space $X$ into a topological space $Y$ is called proper if for every compact subset $K$ of $Y, f^{-1}(K)$ is compact. Following this, given $k \geqslant 0$, we define a mapping $f: \bar{\Omega} \rightarrow F$ to be $k$-proper if $f$ is continuous and $\alpha\left(f^{-1}(S)\right) \leqslant k \alpha(S)$, for each bounded set $S \subseteq F$. By virtue of property 1 of the measure of noncompactness if $f$ is a $k$-proper mapping for some $k>0$, then $f$ is proper.

THEOREM 1.5. Let $f: \bar{\Omega} \rightarrow F$ be $k_{1}$-proper and $h: \bar{\Omega} \rightarrow F$ a $k$-set contraction. Then for each bounded set $S \subseteq F$,

$$
\alpha\left[(f-h)^{-1}(S)\right] \leqslant k_{1} \alpha(S)+k_{1} k \alpha\left[(f-h)^{-1}(S)\right] .
$$

Proof. Let set $(f-h)^{-1}(S)=P$. Let $x \in P$. Then there exists $y \in S$ such that $y=f(x)-h(x)$, that is, $f(x)=y+h(x)$, that is, $x \in f^{-1}(y+h(x))$. Thus it follows that $P \subseteq f^{-1}(S+h(P))$. Hence $\alpha(P) \leqslant \alpha\left[f^{-1}(S+h(P))\right] \leqslant$ $k_{1} \alpha(S+h(P)) \leqslant k_{1}[\alpha(S)+\alpha(h(P))] \leqslant k_{1} \alpha(S)+k_{1} k \alpha(P)$. 
CoRollaRY 1.1. Let $f: \bar{\Omega} \rightarrow F$ be $k_{1}$-proper and $h: \bar{\Omega} \rightarrow F$ be compact (that is, $h$ is continuous and maps each bounded set onto a relatively compact set). Then $(f-h): \bar{\Omega} \rightarrow F$ is $k_{1}$-proper.

PROoF. Since $k=0$, the corollary follows from Theorem 1.5.

Our next theorem will find application in Section 3 and also furnishes an example of a $k$-proper mapping for some $k$.

Let $L: E \rightarrow F$ be a bounded linear Fredholm operator of index zero. Then we can write $E=M \oplus N$ and $F=U \oplus V$ where $N=N(L)=$ the kernel of $L$ and $U=R(L)=$ the range of $L, \operatorname{dim} N=\operatorname{dim} V<\infty$. Let $P: E \rightarrow N$ and $Q$ : $F \rightarrow V$ be continuous projections. Also let $\tilde{L}: M \rightarrow U$ denote the restriction of $L$ to the closed subspace $M$, and $\phi: N \rightarrow V$ be an isomorphism. Then it can easily be seen that $L+\phi P$ is an isomorphism.

THEOREM 1.6. Let $L: E \rightarrow F$ be a bounded linear Fredholm mapping of index zero. Then there exists a linear compact mapping $K$ such that $L+K$ is an isomorphism and is a $k=\left\|\tilde{L}^{-1}\right\|$-proper mapping (in the sense that $\alpha\left((L+K)^{-1}(S)\right) \leqslant k \alpha(S)$ for each bounded set $\left.S \subseteq F\right)$.

Proof. Now $K=\phi P$. Let $S \subseteq F$ be bounded and let $y \in S$. Thus $y=u+v$, where $u \in U$ and $v \in V$. Set $x=(L+K)^{-1} y=m+n$, where $m \in M$ and $n \in N$. Thus $(L+K))(m+n)=u+v$ so $L m+K n=u+v$. Hence $v=K n$ and $u=L m$ so that $(L+K)^{-1}(S) \subseteq \tilde{L}^{-1}((I-Q)(S))+\phi^{-1}(Q(S))$. Thus $\alpha\left((L+K)^{-1}(S)\right) \leqslant \alpha\left(\tilde{L}^{-1}((I-Q)(S))\right)+\alpha\left(\phi^{-1}(Q(S))\right)$. Now $\phi^{-1}(Q(S))$ is compact so $\alpha\left(\phi^{-1}(Q(S))\right)=0$ and $\alpha\left((L+K)^{-1}(S)\right) \leqslant \alpha\left(\tilde{L}^{-1}((I-Q)(S))\right) \leqslant$ $\left\|\tilde{L}^{-1}\right\| \alpha((I-Q)(S))$. It suffices to show that $\alpha((I-Q)(S)) \leqslant \alpha(S)$. Now $(I-Q)(S) \subseteq S-Q(S)$ so $\alpha((I-Q)(S)) \leqslant \alpha(S)+\alpha(Q(S))$ and again since $\bar{Q}(S)$ is compact $\alpha(Q(S))=0$, and the result follows.

REMARK 1.1. From the proof of the above result we have $\alpha(S)=\alpha((I-Q)(S))$, since $S \subseteq(I-Q)(S)+Q(S)$ so $\alpha(S) \leqslant \alpha((I-Q)(S))+\alpha(Q(S))=$ $\alpha((I-Q)(S))$.

\section{Epi mappings}

As we have already indicated, throughout the rest of the paper, unless otherwise stated $E$ and $F$ will denote real Banach spaces and $\Omega$ an open bounded subset of $E$. 
Definition 2.1. A continuous mapping $f: \bar{\Omega} \rightarrow F$ is said to be 0 -admissible ( $p$-admissible) if $f(x) \neq 0(f(x) \neq p \in F)$ for $x \in \partial \Omega$.

Before stating our next definition we recall (Furi, Martelli and Vignoli [1]) that a 0 -admissible mapping $f: \bar{\Omega} \rightarrow F$ is called 0-epi if for each compact mapping $h$ : $\bar{\Omega} \rightarrow F$ with $h(x) \equiv 0$ on $\partial \Omega$ the equation $f(x)=h(x)$ has a solution in $\Omega$. A $p$-admissible mapping $f: \bar{\Omega} \rightarrow F$ is called $p$-epi if the mapping $f-p$ defined by $(f-p)(x)=f(x)-p, x \in \bar{\Omega}$ is 0 -epi.

Definition 2.2. A 0 -admissible mapping $f: \bar{\Omega} \rightarrow F$ is said to be $(0, k)$-epi if for each $k$-set contraction $h: \bar{\Omega} \rightarrow F$ with $h(x) \equiv 0$ on $\partial \Omega$ the equation $f(x)=$ $h(x)$ has a solution in $\Omega$. Similarly a $p$-admissible mapping $f: \bar{\Omega} \rightarrow F$ is said to be $(p, k)$-epi if the mapping $f-p$ defined by $(f-p)(x)=f(x)-p, x \in \bar{\Omega}$, is $(0, k)$-epi.

REMARK 2.1. Since every compact mapping is a $k$-set contraction it follows that every $(0, k)$-mapping is 0 -epi and every $(p, k)$-epi mapping is $p$-epi. In fact, the class of $(p, 0)$-epi mappings is strictly larger than that of $(p, k)$-epi mappings.

A little later we will give an example of a 0 -epi mapping which is not $(0, k)$-epi for some $k$. However, the importance of studying $(p, k)$-epi mappings lies in the fact that a $(p, k)$-epi mapping is in a sense more solvable at the point $p$ than a $p$-epi mappings. This can be made clear by introducing the following terminology.

Measure of unsolvability. Let $f: \bar{\Omega} \rightarrow F$ be a $p$-admissible mapping and $A(f, p)=\{k \geqslant 0:$ there exists a $k$-set contraction $g: \bar{\Omega} \rightarrow F$ with $g \equiv 0$ on $\partial \Omega$ such that the equation $f(x)-p=g(x)$ has no solution in $\Omega$ \}. We define

$$
\gamma(f, p)=j \begin{cases}\inf A(f, p) & \text { if } A(f, p) \neq \varnothing \\ \infty & \text { if } A(f, p)=\varnothing .\end{cases}
$$

We call $\gamma(f, p)$ the measure of unsolvability of $f$ at $p$. Thus $\gamma(f, p): A(\bar{\Omega}, p) \rightarrow$ $[0, \infty]$ is a well defined mapping, where $A(\bar{\Omega}, p)$ is the set of all $p$-admissible mapping $f: \bar{\Omega} \rightarrow F$. It then follows from the above definition that for a $(p, 0)$-epi mapping $f: \bar{\Omega} \rightarrow F, \gamma(f, p) \geqslant 0$ while $\gamma(f, p) \geqslant k$ for a $(p, k)$-epi mapping $f$ : $\bar{\Omega} \rightarrow F$.

We recall that a mapping of the form $I-h$ is called a compact vector field where $h: \bar{\Omega} \rightarrow E$ is a compact mapping and $I$ is the identity on $E$. (See Granas [4].)

LemMA 2.1. Let $(I-h): \bar{\Omega} \rightarrow E$ be a compact vector field and assume that $h \equiv 0$ on $\partial \Omega$. Then $\gamma(f, p) \geqslant 1$ if and only if $p \in \Omega$, where $f=I-h$. 
Proof. First note that $\gamma(f, p)$ is defined only when $f$ is $p$-admissible, that is when $f(x)=x-h(x) \neq p$ for all $x \in \partial \Omega$. This implies that $p \notin \partial \Omega$ as $h \equiv 0$ on $\partial \Omega$. Thus $p \in E \backslash \partial \Omega$.

Now suppose that $p \in \Omega$ and let $g: \bar{\Omega} \rightarrow E$ be a $k$-set contraction with $0 \leqslant k<1$ and $g \equiv 0$ on $\partial \Omega$. Setting $l(x)=p+h(x)+g(x), x \in \bar{\Omega}$ we see that $l$ is a $k$-set contraction. We define $\bar{l}: E \rightarrow E$ by

$$
l(x)= \begin{cases}l(x) & \text { if } x \in \bar{\Omega}, \\ p & \text { if } x \notin \bar{\Omega} .\end{cases}
$$

By Theorem 1.3, $\bar{l}$ is a $k$-set contraction and therefore $\bar{l}(\bar{\Omega})$ is bounded. Let $M=\sup \{\|\bar{l}(x)\|: x \in \bar{\Omega}\}$ and $B=\{x \in E:\|x\| \leqslant M\}$. Then the restriction of $\bar{h}$ to the closed convex ball $B$ is a selfmapping and remains a $k$-set contraction. Hence by Theorem 1.4 there exists a point $x_{0} \in B$ such that $l\left(x_{0}\right)=x_{0}$. Now $x_{0} \in \Omega$, for otherwise $\bar{l}\left(x_{0}\right)=p=x_{0}$ which will contradict the fact that $p \in \Omega$. Thus $x_{0} \in \Omega$ and $x_{0}=l\left(x_{0}\right)=p+h\left(x_{0}\right)+g\left(x_{0}\right)$, that is $f\left(x_{0}\right)-p=g\left(x_{0}\right)$ as required. Next suppose that $\gamma(f, p) \geqslant 1$. Let $g=-h$. Then $g$ is a 0 -set contraction and therefore $f\left(x_{0}\right)-p-g\left(x_{0}\right)=0$ for some $x_{0} \in \Omega$. Hence $p=x_{0} \in \Omega$, as required.

EXAMPLE 2.1. More generally, let $h: \bar{\Omega} \rightarrow E$ be a $k$-set contraction such that $k \in[0,1)$ and $p \notin(I-h)(\partial \Omega)$. We also assume that the (Nussbaum [9]) degree, $d(f, \Omega, p) \neq 0$ where $f=I-h$. We choose $\varepsilon>0$ sufficiently small that $k+\varepsilon<1$ and consider a $[1-(k+\varepsilon)]$-set contraction $g: \bar{\Omega} \rightarrow E$ such that $g \equiv 0$ on $\partial \Omega$. Then clearly $h+g$ is a $(1-\varepsilon)$-set contraction and $h+g \equiv h$ on $\partial \Omega$. Hence by the boundary dependence property of the Nussbaum degree $d(I-h, \Omega, p)=$ $d(I-(h+g), \Omega, p) \neq 0$. Hence by the existence property of the degree there exists a solution $x_{0} \in \Omega$ of the equation $f(x)-p=g(x)$, that is $\gamma(f, p) \geqslant 1-k$.

EXAMPLE 2.2. Now we give an example where $d(f, \Omega, 0)=0$ but $f$ is $(0, k)$-epi, for all $k$. Let $E=\mathbf{R}$, the real line, $\Omega=(-2,0) \cup(0,2)$ and $f: \bar{\Omega} \rightarrow E$ be defined by $f(x)=x^{2}-1$. Clearly $d(f, \Omega, 0)=0$ but $\gamma(f, 0)=\infty$. To see that $\gamma(f, 0)=$ $\infty$ let $g: \bar{\Omega} \rightarrow E$ be continuous and let $g \equiv 0$ on $\partial \Omega$. Now we note that $d\left(\left.f\right|_{[0,2]}\right.$, $(0,2), 0)=1$. Since $f-g=f$ on $\partial((0,2))$ we have $d\left(f-\left.g\right|_{[0,2]},(0,2), 0\right)=1$. The conclusion follows from the definition of $\gamma$.

We now return to $(p, k)$-epi mappings and prove some of their basic properties.

EXISTENCE PROPERTY. If $f: \bar{\Omega} \rightarrow F$ is a ( $p, k)$-epi mapping, then the equation $f(x)=p$ has a solution in $\Omega$. 
Proof. Using $h(x) \equiv 0$ on $\bar{\Omega}$ in the definition of $(p, k)$-epi, we obtain a solution of the equation $f(x)=p$ in $\Omega$.

NORMALIZATION PROPERTY. The inclusion mapping $i: \bar{\Omega} \rightarrow E$ is $(p, k)$-epi for $k \in[0,1)$ if and only if $p \in \Omega$, that is, $\gamma(i, p) \geqslant 1$ if and only if $p \in \Omega$.

This is a special case of Lemma 2.1 with $h \equiv 0$ in $\bar{\Omega}$.

LOCALIZATION PROPERTY. If $f: \bar{\Omega} \rightarrow F$ is $(0, k)$-epi and $f^{-1}(0)$ is contained in an open set $\Omega_{1} \subset \Omega$, then $f$ restricted to $\Omega_{1}$ is also $(0, k)$-epi.

Proof. Let $h: \Omega_{1} \rightarrow F$ be a $k$-set contraction such that $h \equiv 0$ on $\partial \Omega_{1}$. Define $\bar{h}: E \rightarrow F$ by

$$
\bar{h}(x)= \begin{cases}h(x) & \text { if } x \in \bar{\Omega}_{1}, \\ 0 & \text { if } x \notin \bar{\Omega}_{1} .\end{cases}
$$

Then $\bar{h}$ is, by Theorem 1.3 , a $k$-set contraction and $h_{1}$, the restriction of $\bar{h}$ to $\bar{\Omega}$, is, therefore, a $k$-set contraction and obviously $h_{1} \equiv 0$ on $\partial \Omega$. Thus the equation $f(x)=h_{1}(x)$ has a solution $x_{0} \in \Omega$. Now $f^{-1}(0) \subseteq \Omega_{1}$ implies that $x_{0} \in \Omega_{1}$. Therefore $f(x)=h(x)$ has a solution $x_{0} \in \Omega_{1}$, as required

HOMOTOPY PROPERTY. Let $f: \bar{\Omega} \rightarrow F$ be $(0, k)$-epi and $h:[0,1] \times \bar{\Omega} \rightarrow F$ be an $\alpha$-set contraction with $0 \leqslant \alpha \leqslant k<1$ such that $h(0, x)=0$ for all $x \in \bar{\Omega}$. Further let $f(x)+h(t, x) \neq 0$ for all $x \in \partial \Omega$ and for all $t \in[0,1]$. Then $f(\cdot)+h(1, \cdot)$ : $\overline{\mathbf{\Omega}} \rightarrow F$ is $(0, k-\alpha)$-epi.

Proof. Let $g: \bar{\Omega} \rightarrow F$ be a $(k-\alpha)$-set contraction such that $g \equiv 0$ on $\partial \Omega$. The set $S=\{x \in \bar{\Omega}: f(x)+h(t, x)=g(x)$ for some $t \in[0,1]\}$ is a closed set since $f, g, h$ are continuous and $[0,1]$ is compact. Note that $g$ being a $(k-\alpha)$-set contraction is a $k$-set contraction. Hence there exists $x_{0} \in \Omega$ such that $f\left(x_{0}\right)=$ $g\left(x_{0}\right)$. Thus $f\left(x_{0}\right)+h\left(0, x_{0}\right)=g\left(x_{0}\right)$ and $S$ is nonempty. Moreover $S$ and $\partial \Omega$ are disjoint. Hence by Urysohn's Lemma there exists a continuous function $\phi$ : $\bar{\Omega} \rightarrow[0,1]$ such that $\phi \equiv 1$ on $S$ and $\phi \equiv 0$ on $\partial \Omega$. We now consider the function $\bar{h}: \bar{\Omega} \rightarrow F$ defined by $\bar{h}(x)=g(x)-h(\phi(x), x), x \in \bar{\Omega}$. In view of Theorem 1.1 $\bar{h}$ is a $k$-set contraction. Also $\bar{h} \equiv 0$ on $\partial \Omega$. Hence the equation $f(x)=\bar{h}(x)=$ $g(x)-h(\phi(x), x)$ has a solution $x_{0} \in \Omega$. This implies $x_{0} \in S$. Hence $f(x)+$ $h(1, x)=g(x)$ has a solution $x_{0} \in \Omega$.

Thus $\gamma(\cdot, p)$ has the essential features of a degree theory. 
BOUNDARY DEPENDENCE PROPERTY. Let $f: \bar{\Omega} \rightarrow F$ be $(0, k)$-epi and $g: \bar{\Omega} \rightarrow F$ be an $\alpha$-set contraction with $0 \leqslant \alpha \leqslant k<1$ and $g \equiv 0$ on $\partial \Omega$. Then $(f+g)$ : $\bar{\Omega} \rightarrow F$ is $(0, k-\alpha)$-epi.

Proof. Let $h$ be a $(k-\alpha)$-set contraction and $h \equiv 0$ on $\partial \Omega$. Then $(h-g)$ : $\bar{\Omega} \rightarrow F$ is a $k$-set contraction and $(h-g) \equiv 0$ on $\partial \Omega$. Hence $f(x)=(h-g)(x)$, that is, $f(x)+g(x)=h(x)$ has a solution in $\Omega$.

We now consider the $k$-proper mapping which we have introduced in Section 1. Since every $k$-proper mapping $f: \bar{\Omega} \rightarrow F$ is proper, it follows that if $f: \bar{\Omega} \rightarrow F$ is $k$-proper, then $f(\bar{\Omega})$ is closed and hence $f(\bar{\Omega})=\overline{f(\Omega)}$, by continuity of $f$.

THEOREM 2.1. If $f: \bar{\Omega} \rightarrow F$ is $(0, k)$-epi with $k \in[0,1)$ and proper, then $f$ maps $\Omega$ onto a neighborhood of the origin. More generally, if $U$ is the connected component of $F \backslash f(\partial \Omega)$ containing the origin, then $U \subset f(\Omega)$.

Proof. Since $f$ is proper, $f(\partial \Omega)$ is closed. Thus $U$ is an open set and is path connected. Assume that $p \in U$ and $\phi:[0,1] \rightarrow U$ is a continuous mapping with $\phi(0)=0$ and $\phi(1)=p$. Then taking the 0 -set contraction $h(x, t)=-\phi(t)$ in our homotopy property, we see that $f(\cdot)-\phi(1): \bar{\Omega} \rightarrow F$ is $(0, k)$-epi. Thus $f(x)-$ $\phi(1)=0$ has a solution in $\Omega$, that is, $p=\phi(1) \in f(\Omega)$.

In the following theorem by a $\left(p, 1^{-}\right)$-epi we mean a $p$-admissible mapping $f$ : $\bar{\Omega} \rightarrow F$ such that for each condensing mapping $f: \bar{\Omega} \rightarrow F$ with $g \equiv 0$ on $\partial \Omega$, the equation $f(x)-p=g(x)$ has a solution in $\Omega$.

THEOREM 2.2. Let $f: \bar{\Omega} \rightarrow F$ be continuous, injective and 1-proper. T.:en $f(\Omega)$ is open if and only if $f$ is $\left(p, 1^{-}\right)$-epi for any $p \in f(\Omega)$.

Proof. Let $p \in f(\Omega)$. Since $f$ is proper, it follows from Theorem 2.1 that $f-p$ being also $(0,0)$-epi maps $\Omega$ onto a neighbourhood of 0 . Thus $f(\Omega)$ is open.

Now let $f(\Omega)$ be open. It suffices to prove that if $0 \in f(\Omega)$ then $f$ is $\left(0,1^{-}\right)$-epi. So we assume $0 \in f(\Omega)$. Now since $f(\Omega)$ is open, $f$ is injective and 1-proper and hence proper, $f^{-1}$ is continuous. Also since $\overline{f(\Omega)}=f(\bar{\Omega})$, we have $\partial(f(\Omega))=$ $f(\partial \Omega)$. Let $h: \bar{\Omega} \rightarrow F$ be condensing such that $h \equiv 0$ on $\partial \Omega$.

We define $g: F \rightarrow F$ by

$$
g(y)= \begin{cases}h\left(f^{-1}(y)\right), & y \in \overline{f(\Omega)} \\ 0, & \text { otherwise }\end{cases}
$$


Since $\partial(f(\Omega))=f(\partial \Omega), g \equiv 0$ on $\partial(f(\Omega))$. Thus it follows that $g$ is continuous. Now let $S$ be a bounded subset of $F$. Then $\alpha[g(S)]=\alpha\left[h\left(f^{-1}\{S \cap f(\bar{\Omega})\}\right)\right] \leqslant$ $\alpha\left[f^{-1}\{S \cap f(\bar{\Omega})\}\right] \leqslant \alpha(S \cap f(\bar{\Omega})) \leqslant \alpha(S)$. Also since $g(F)=g(\overline{f(\Omega)}) \cup\{0\}=$ $g(f(\bar{\Omega})) \cup\{0\}=h(\bar{\Omega}) \cup\{0\}$ and $h$ is condensing, $g(F)$ is bounded. Let $M=$ $\sup \{\|y\|: y \in g(F)\}$. Let $B=\{x \in F:\|x\| \leqslant M\}$. Then $g$ restricted to $B$ maps $B$ into itself and is, by what we have proved above, a condensing mapping. Hence by Theorem 1.4 there is a point $y_{0} \in B$ such that $g\left(y_{0}\right)=y_{0}$. Since $0 \in f(\Omega)$, it follows that $y_{0} \in f(\Omega)$. Hence $x_{0}=f^{-1}\left(y_{0}\right) \in \Omega$ is a solution of the equation $f(x)=h(x)$.

THEOREM 2.3. Let $f: \bar{\Omega} \rightarrow F$ be continuous, injective and $k_{1}$-proper. Then $f(\Omega)$ is open if and only if $f$ is $(p, k)$-epi for each $p \in f(\Omega)$ and each nonnegative $k$ satisfying the condition $k_{1} k<1$.

Proof. The argument for the 'if part' is the same as in the proof of Theorem 2.2. For the 'only if part' let $h: \bar{\Omega} \rightarrow F$. be a $k$-set contraction. The rest of the argument will be the same as in the proof of Theorem 2.2 except that we need to show that the mapping $g$ as constructed there is a $k_{1} k$-set contraction. Indeed $\alpha[g(S)]=\alpha\left[h\left(f^{-1}\{S \cap f(\bar{\Omega})\}\right)\right] \leqslant k \alpha\left[f^{-1}\{S \cap f(\bar{\Omega})\}\right] \leqslant k k_{1} \alpha\{S \cap f(\bar{\Omega})\} \leqslant$ $k k_{1} \alpha(S)$.

COROLlaRY 2.1. Let $f: \bar{\Omega} \rightarrow F$ be continuous, injective and $k_{1}$-proper and $f(\Omega)$ be open. Then $f$ is $(p, k)$-epi for each nonnegative $k$ satisfying $k_{1} k<1$ if and only if $p \in f(\Omega)$.

Proof. If $f$ is $(p, k)$-epi, then $p \in f(\Omega)$ by the existence property (regardless of whether $f(\Omega)$ is open or not). If $f(\Omega)$ is open, the result follows from the Theorem 2.3.

THEOREM 2.4. Let $f: \bar{\Omega} \rightarrow F$ be continuous, injective and proper. Then $f(\Omega)$ is open if and only if $f$ is $(p, 0)$-epi for every $p \in f(\Omega)$.

Proof. Again the argument is the same as in the proof of Theorem 2.2, except that we use the fact that $g(F)=h(\bar{\Omega}) \cup\{0\}$ is relatively compact as $h$ is a compact mapping. The fixed point of $\left.g\right|_{B}: B \rightarrow B$ is guaranteed by the Schauder fixed point theorem.

The above theorem and following corollary have been obtained by Furi, Martelli and Vignoli [1]. 
CoRollary 2.2. Let $f: \bar{\Omega} \rightarrow F$ be continuous, injective and proper and $f(\Omega)$ open. Then $f$ is $(p, 0)$-epi if and only if $p \in f(\Omega)$.

Proof. The proof is similar to that of Corollary 2.1, except that we use Theorem 2.4 in place of Theorem 2.3.

REMARK 2.1. For each $k \geqslant 0$, we construct a mapping $f_{k}$ such that $\gamma\left(f_{k}, 0\right)=k$.

We consider the Hilbert space $H=\left\{x=\sum \alpha_{i} e_{i}:-\infty<i<\infty, \sum \alpha_{i}^{2}<\infty\right\}$ where $\left\{e_{i}\right\}$ is the orthonormal basis, that is, $\left\langle e_{i}, e_{j}\right\rangle=0$ if $i \neq j$ and $=1$ if $i=j$. The norm of $H$ is defined by $\|x\|=\left|\sum \alpha_{i}^{2}\right|^{1 / 2}$, where $x=\sum \alpha_{i} e_{i}$.

Let $\Omega=B(0,1)=\{x \in H:\|x\|<1\}$. Let $f: \bar{\Omega} \rightarrow H$ be defined by $f(x)=$ $(1 / 2 d) x, x \in \bar{\Omega}$ where $d>0$ is a constant. Clearly $f: \bar{\Omega} \rightarrow H$ is continuous, injective and $2 d$-proper and $f(\Omega)$ is open as $f$ is a homomorphism onto $f(\bar{\Omega})$ $(=\overline{f(\Omega)})$. Hence, by Corollary $2.1, f$ is $(p, k)$-epi for each nonnegative $k<1 / 2 d$ and each $p \in f(\Omega)$, that is, $\gamma(f, p) \geqslant 1 / 2 d$ for each $p \in f(\Omega)$. We now construct a $\bar{k}$-set contraction $h: \bar{\Omega} \rightarrow H$ for some positive number $\bar{k}$, with $h \equiv 0$ on $\partial \Omega$ such that $f(x)=h(x)$ has no solution in $\Omega$, that is, $\gamma(f, 0) \leqslant \bar{k}$.

Let $u: \bar{\Omega} \rightarrow H$ be defined by $u(x)=\sum \alpha_{i} e_{i+1}$ for $x=\sum \alpha_{i} e_{i} \in \bar{\Omega}$ and $\phi:$ $\bar{\Omega} \rightarrow H$ by

$$
\phi(x)=(1-\|x\|) u(x)+\|x\| x+\frac{1}{3}(1-\|x\|) e_{0}, \quad x \in \bar{\Omega} .
$$

Finally we defined $h: \bar{\Omega} \rightarrow H$ by $h(x)=(x-\phi(x)) / d, x \in \bar{\Omega}$. It is easy to verify that $h \equiv 0$ on $\partial \Omega$. We next verify that $h$ is a $k$-set contraction for some positive number $\bar{k}$. On simplifying $h(x)=\frac{1}{d}(1-\|x\|)\left(x-u(x)-\frac{1}{3} e_{0}\right)$. Hence for $x, y \in \bar{\Omega}$,

$$
\begin{gathered}
h(x)-h(y)=\frac{1}{d}\left[(1-\|x\|)\left(x-u(x)-\frac{1}{3} e_{0}\right)-(1-\|y\|)\left(y-u(y)-\frac{1}{3} e_{0}\right)\right] \\
=\frac{1}{d}\left[(1-\|x\|)\{(x-u(x))-(y-u(y))\}-\left\{\left(y-u(y)-\frac{1}{3} e_{0}\right)\right\}\right. \\
\times(\|x\|-\|y\|)] \\
=\frac{1}{d}\left[(1-\|x\|)(v(x)-v(y))-\left(v(y)-\frac{1}{3} e_{0}\right)(\|x\|-\|y\|)\right]
\end{gathered}
$$

where $v=I-u: H \rightarrow H$ is a bounded linear operator on $H$.

Hence

$$
\begin{aligned}
\|h(x)-h(y)\| & \leqslant \frac{1}{d}\left[\mid 1-\|x\|\|v\|\|x-y\|+\left(\|v\|\|y\|+\frac{1}{3}\right)(\|x\|-\|y\|)\right] \\
& \leqslant \frac{1}{d}\left[\|v\|+\|v\|+\frac{1}{3}\right]\|x-y\| .
\end{aligned}
$$

Thus $h$ is $\bar{k}$-set contraction where $\bar{k}=\left(2\|v\|+\frac{1}{3}\right) \frac{1}{d}$. Next we show that $f(x)=$ $h(x)$ has no solution in $\bar{\Omega}$. Suppose that $f\left(x_{0}\right)=h\left(x_{0}\right)$ for some $x_{0} \in \bar{\Omega}$. 
Case 1. Let $x_{0} \in \partial \Omega$ an $f\left(x_{0}\right)=h\left(x_{0}\right)$, then $x_{0} / 2 d=0$ by definition of $f$ and $h$, which is a contradiction. Hence $\left\|x_{0}\right\|<1$.

Case 2. Let $\left\|x_{0}\right\|=\frac{1}{2}$. Then $x_{0}=2 \phi\left(x_{0}\right)$, that is, $x_{0}=u\left(x_{0}\right)+x_{0}+\frac{1}{3} e_{0}$, that is, $u\left(x_{0}\right)+\frac{1}{3} e_{0}=0$, that is $\sum \alpha_{i+1} e_{i}+\frac{1}{3} e_{0}=0$, where $x_{0}=\sum \alpha_{i} e_{i}$. This implies that $\alpha_{1}=-\frac{1}{3}$, and $\alpha_{j}=0$ for $j \neq 1$. Thus $\left\|x_{0}\right\|=\frac{1}{3}$ which is a contradiction. Hence $\left\|x_{0}\right\|=t<1$ and $t \neq \frac{1}{2}$.

Case 3. Let $\left\|x_{0}\right\|=t<1$ and $t \neq \frac{1}{2}$. As $\phi(0) \neq 0$ and $x_{0}=2 \phi\left(x_{0}\right)$, we have $x_{0}=2(1-t) u\left(x_{0}\right)+2 t x_{0}+\frac{2}{3}(1-t) e_{0}$, that is, $\sum \alpha_{i} e_{i}=2(1-t) \sum \alpha_{i} e_{i+1}+$ $2 t \sum \alpha_{i} e_{i}+\frac{2}{3}(1-t) e_{0}$ where $x_{0}=\sum \alpha_{i} e_{i}$. Hence $(1-2 t)(1-t)^{-1} \sum \alpha_{i} e_{i}=$ $2 \sum \alpha_{i} e_{i+1}+\frac{2}{3} e_{0}$. Thus $(1-\delta) \sum \alpha_{i} e_{i}=2 \sum \alpha_{i} e_{i+1}+\frac{2}{3} e_{0}$, where $\delta=t /(1-t) \neq 1$ as $t \neq \frac{1}{2}$. Hence $(1-\delta) \alpha_{i+1}=2 \alpha_{i}+\frac{2}{3} \delta_{0, i+1}$ where

$$
\delta_{i, j}= \begin{cases}0 & \text { if } i \neq j, \\ 1 & \text { if } i=j,\end{cases}
$$

and $1-\delta \neq 0$. Hence $\left|\alpha_{j}\right| \rightarrow \infty$ as $j \rightarrow \infty$ or $\left|\alpha_{j}\right| \rightarrow \infty$ as $j \rightarrow-\infty$ according as $2 /(1-\delta)>1$ or $2 /(1-\delta)<1$. But $\sum \alpha_{i}^{2}<\infty$ which is a contradiction. Thus we conclude that $h\left(x_{0}\right) \neq f\left(x_{0}\right)$ for any $x_{0} \in \bar{\Omega}$. Hence $f$ is not $(0, \bar{k})$-epi and hence $\gamma(f, 0) \leqslant \bar{k}$. Thus there exists $r$ such that $(2 d)^{-1} \leqslant r \leqslant \bar{k}$ and $f$ is $(0, s)$-epi for each $s<r$ but not $(0, s)$-epi for $s>r$. Setting $f_{k}=k f / r$, the result follows.

We recall that a set $Q \subset F$ is said to be star-shaped with respect to the origin if ty $\in Q$ whenever $y \in Q$ and $t \in[0,1]$.

TheOREM 2.5. Let $f: \bar{\Omega} \rightarrow F$ be $(0, k)$-epi with $k \in[0,1)$ and $Q$ a star-shaped subset of $F$ with respect to the origin such that $Q \cap f(\partial \Omega)=\varnothing$. Then for each $k_{1}$ set contraction $h: \bar{\Omega} \rightarrow F$ with $h(\partial \Omega) \subset Q$ and $0 \leqslant k_{1} \leqslant k<1$, the equation $f(x)=h(x)$ has solution in $\Omega$. In particular $Q \subset f(\Omega)$.

Proof. It is clear that for all $x \in \partial \Omega$ and for all $t \in[0,1], t h(x) \in Q$ and $f(x) \notin Q$. Hence $f(x) \neq t h(x)$ for all $x \in \partial \Omega$ and for all $t \in[0,1]$. We define the mapping $H:[0,1] \times \bar{\Omega} \rightarrow F$ by $H(t, x)=-t h(x)$, for all $(t, x) \in[0,1] \times \bar{\Omega}$. Clearly for each fixed $t \in[0,1]$, the mapping $H_{t}: \bar{\Omega} \rightarrow F$ defined by $H_{t}(x)=$ $H(t, x)=-t h(x)$ is a $k_{1}$-set contraction $\left(\right.$ as $\alpha\left(H_{t}(S)\right)=\alpha(t h(S))=t \alpha(h(S)) \leqslant$ $t k_{1} \alpha(S) \leqslant k_{1} \alpha(S)$, for every set $\left.S \subset \bar{\Omega}\right)$. Hence by Theorem $1.2, H:[0,1] \times \bar{\Omega} \rightarrow F$ is a $k_{1}$-set contraction. Also $H(0, x)=0$ for all $x \in \bar{\Omega}$. Thus by the homotopy property $f(\cdot)+H(1, \cdot)=f(\cdot)-h(\cdot)$ is $\left(0, k-k_{1}\right)$-epi. Hence $f(x)=h(x)$ has a solution in $\Omega$. Now let $p \in Q$. The constant mapping $h(x)=p$ for all $x \in \bar{\Omega}$ is a 0 -set contraction. Hence by the above $f(x)=p$ for some $x \in \Omega$. Thus $Q \subset f(\Omega)$. 
THEOREM 2.6. Let $f: \bar{\Omega} \rightarrow F$ be continuous, 0 -admissible and $k_{1}$-proper and let $\left\{f_{n}: f_{n}: \bar{\Omega} \rightarrow F\right\}$ be a sequence of $(0-k)$-epi mappings with $k_{1} k<1$ such that $f_{n} \rightarrow$ f uniformly. Then $f$ is $(0, k)$-epi.

Proof. Let $h: \bar{\Omega} \rightarrow F$ be a $k$-set contraction such that $h \equiv 0$ on $\partial \Omega$. For each $n=1,2, \ldots$, there exists $x_{n} \in \Omega$ such that $f_{n}\left(x_{n}\right)=h\left(x_{n}\right)$. Let $y_{n}=f\left(x_{n}\right)-$ $f_{n}\left(x_{n}\right)=f\left(x_{n}\right)-h\left(x_{n}\right)$. Then $y_{n} \rightarrow 0$ as $n \rightarrow \infty$. Let set $S=\left\{y_{n}: n=1,2, \ldots\right\}$. Then by Theorem 1.5,

$$
\alpha\left[(f-h)^{-1}(S)\right] \leqslant k_{1} k \alpha\left[(f-h)^{-1}(S)\right] \text { as } \alpha(S)=0 .
$$

Now as $k_{1} k<1$, this implies that $(f-h)^{-1}(S)$ is relatively compact. Hence $\left\{x_{n}\right.$ : $n=1,2, \ldots\}$ being a subset of $(f-h)^{-1}(S)$ must have a limit point $x_{0}$. Clearly $f\left(x_{0}\right)=h\left(x_{0}\right)$.

THEOREM 2.7 (Perturbation). Let $f: \bar{\Omega} \rightarrow F$ be $(0, k)$-epi and $k_{1}$-proper and let $h:[0,1] \times \bar{\Omega} \rightarrow F$ be a $k_{2}$-set contraction such that $0 \leqslant k_{2} \leqslant k<1$ and $h(0, x)=0$ for all $x \in \bar{\Omega}$. If $k_{1} k_{2}<1$, then there exists $\varepsilon>0$ such that $f(\cdot)-h(t, \cdot)$ is $\left(0, k-k_{2}\right)$-epi for all $t$ satisfying $|t|<\varepsilon$.

Proof. In view of the homotopy property, it will suffice to show that there exists $\varepsilon>0$ such that $f(x)-h(t, x) \neq 0$ for all $x \in \partial \Omega$ and for all $t \in(-\varepsilon, \varepsilon)$. We assume that there is no such $\varepsilon>0$. Then we can show that there exists a sequence $\left\{\left(t_{n}, x_{n}\right)\right\}$ with $t_{n} \rightarrow 0, x_{n} \in \partial \Omega$ and $f\left(x_{n}\right)=h\left(t_{n}, x_{n}\right)=y_{n}$, for all $n$. Now using the fact that $f$ is $k_{1}$-proper and $h$ is a $k_{2}$-set contraction,

$$
\begin{aligned}
\alpha\left[x_{n}: n\right. & =1,2, \ldots] \leqslant \alpha\left[f^{-1}\left(y_{n}\right): n=1,2, \ldots\right] \leqslant k_{1} \alpha\left[y_{n}: n=1,2, \ldots\right] \\
& =k_{1} \alpha\left[h\left(t_{n}, x_{n}\right): n=1,2, \ldots\right] \leqslant k_{1} k_{2} \alpha\left[\left(t_{n}, x_{n}\right): n=1, \ldots, \ldots\right] \\
& \leqslant k_{1} k_{2} \alpha\left[\left\{t_{n}\right\} \times\left\{x_{m}\right\}: m, n=1,2, \ldots\right]=k_{1} k_{2} \alpha\left[x_{m}: m=1,2, \ldots\right],
\end{aligned}
$$

by Lemma 1.1. Since $k_{1} k_{2}<1, \alpha\left[x_{n}: n=1,2, \ldots\right]=0$. Hence the sequence $\left\{x_{n}\right\}$ has a limit point $x_{0}$. It follows that $f\left(x_{0}\right)=h\left(0, x_{0}\right)=0$ which contradicts the fact that $f$ is 0 -admissible.

If $f: \bar{\Omega} \rightarrow E$ is a $k$-set contraction mapping such that $k \in[0,1), I$ is the identity mapping on $E$, and $p \notin(I-f)(\partial \Omega)$, then the degree of $I-F$ at $p$, $d(I-f, \Omega, p)$ is well defined (see [7], p. 95). The following theorem is similar to the example following the definition of measure of unsolvability.

THEOREM 2.8. Let $I-f: \bar{\Omega} \rightarrow E$ be $p$-admissible and let $f: \bar{\Omega} \rightarrow E$ be a $k$-set contraction with $k \in[0,1)$. If $d(I-f, \Omega, p) \neq 0$, then for every $k_{1}$ with $0 \leqslant k \leqslant$ $k_{1}<1, I-f$ is $\left(p, k_{1}-k\right)$-epi. 
Proof. Let $h: \bar{\Omega} \rightarrow E$ be a $\left(k_{1}-k\right)$-set contraction such that $h \equiv 0$ on $\partial \Omega$. $(I-(f+h))$ is a $k_{1}$-set contraction and clearly $f+h \equiv f$ on $\partial \Omega$. Hence by the boundary dependence property of the degree, $d(I-(f+h), \Omega, p)=$ $d(I-f, \Omega, p) \neq 0$. Hence there exists $x_{0} \in \Omega$ such that $x_{0}-(f+h)\left(x_{0}\right)=p$. Thus $x_{0}-f\left(x_{0}\right)=p+h\left(x_{0}\right)$, as required.

THEOREM 2.9. Let $L: E \rightarrow F$ be bounded, linear and subjective with $\operatorname{dim} \operatorname{Ker} L$ $<\infty$. Let $g: \bar{\Omega} \rightarrow \mathbf{R}^{n}$ be continuous with bounded range. Let $g(x) \neq 0$ for all $x \in \partial \Omega \cap \operatorname{Ker} L$ and $J: \mathbf{R}^{n} \rightarrow E$ be a linear mapping with $\operatorname{Im} J=\operatorname{Ker} L$. If the Brouwer topological degree $d\left(g J, J^{-1}(\Omega), 0\right) \neq 0$, then $\gamma(M, 0) \geqslant\left\|\tilde{L}^{-1}\right\|$ where $E=\operatorname{Ker} L \oplus N, \tilde{L}$ is the restriction of $L$ to $N$, and $M: \bar{\Omega} \rightarrow F \times \mathbf{R}^{n}$ is defined by $M(x)=(L x, g(x))$.

Proof. The proof is similiar to that of Theorem 1.7 of Furi, Martelli and Vignoli [1]. Let $S=\tilde{L}^{-1}$. We define $A: F \times \mathbf{R}^{n} \rightarrow E$ by $A(y, z)=S y+J z$. Evidently $A$ is an isomorphism. We now consider the mapping $f=M A: A^{-1}(\Omega)$ $\rightarrow F \times \mathbf{R}^{n}$. Then as $S$ is a right inverse of $L$ and $\operatorname{Im} J=\operatorname{Ker} L, f(y, z)=$ $(L(S y+J z), \quad g(S y+J z))=(y, g(S y+J z))=(y, z)-(0, z-g(S y+J z))=$ $(I-h)(y, z)$ where $h: A^{-1}(\Omega) \rightarrow\{0\} \times \mathbf{R}^{n}$ is the mapping defined by $h(y, z)=$ $(0, z-g(S y+J z))$. By using the properties of Leray-Schauder degree

$$
\begin{aligned}
d\left(f, A^{-1}(\Omega), 0\right) & =d\left(\left.f\right|_{\{0\} \times \mathbf{R}^{n}}, A^{-1}(\Omega) \cap\left(\{0\} \times \mathbf{R}^{n}\right), 0\right) \\
& =d\left(g J, J^{-1}(\Omega), 0\right) \neq 0 .
\end{aligned}
$$

Hence by Example 2.1, $\gamma(f, 0) \geqslant 1$. Now $M=f A^{-1}$ and $A^{-1}$ is $\left\|\tilde{L}^{-1}\right\|$ proper and hence $\gamma(M, 0) \geqslant \gamma(f, 0)\left\|\tilde{L}^{-1}\right\|$.

EXAMPLE 2.3. A natural question which arises from Theorem 2.9 is the following. In Theorem 2.9 is it legitimate to replace the assumption that the Brouwer degree $d\left(g J, J^{-1}(\Omega), 0\right) \neq 0$ merely by the assumption that $g J: \overline{J^{-1}(\Omega)}$ $\rightarrow F$ is 0 -epi. This is not legitimate as the following example shows.

Let $\Omega=\left\{(x, y) \in \mathbf{R}^{2}: 0<x^{2}+y^{2}<4\right\} \subseteq E=\mathbf{R}^{2}$ and let $J: \mathbf{R} \rightarrow E$ be given by $J(x)=(x, 0)$ so that $J^{-1}(\Omega)=(-2,0) \cup(0,2)$ Let $g: \bar{\Omega} \rightarrow F=\mathbf{R}$ be given by $g(x, y)=x^{2}+y^{2}-1$. Thus $g J:[-2,2] \rightarrow \mathbf{R}$ is given by $g J(x)=x^{2}-1$ so $g J$ is 0-epi on $\overline{(-2,0) \cup(0,2)}=\overline{J^{-1}(\Omega)}$ from Example 2.2. Let $L: E \rightarrow F$ be given by $L(x, y)=y$. Thus $\operatorname{Im} J=\operatorname{Ker} L$. Let $M(x, y)=\left(y, x^{2}+y^{2}-1\right)=$ $(L(x, y), g(x, y))$. We show that $M: \bar{\Omega} \rightarrow F \times \mathbf{R}$ is not 0 -epi. Define $h: \bar{\Omega} \rightarrow F$ $\times \mathbf{R}$ by $h(x, y)=\left(2\left(x^{2}+y^{2}\right)\left(x^{2}+y^{2}-2\right), 0\right)$. Thus $h(x, y)=(0,0)$ for all $(x, y) \in \partial \Omega, h$ is continuous on $\bar{\Omega}$, and it suffices to show that $M(x, y) \neq h(x, y)$ for all $(x, y) \in \Omega$. Suppose there is a solution $(x, y) \in \Omega$, then $g(x, y)=0$ so $x^{2}+y^{2}=1$. Thus $h(x, y)=-2 \neq L(x, y)=y$ since $|y| \leqslant 1$. 


\section{3. $(p, k)$-epi mappings on the whole space}

As before, $E$ and $F$ are real Banach spaces. Let $f: E \rightarrow F$ be a continuous mapping of $E$ into $F$. For $p \in F, f$ is said to be $p$-admissible if $f^{-1}(p)$ is a bounded subset of $E$. $f$ is said to be $(p, k)$-epi if $f$ is $(p, k)$-epi on the closure of any bounded open set $\Omega \supset f^{-1}(p)$, that is, $\left.f\right|_{\bar{\Omega}}$, the restriction of $f$ to $\bar{\Omega}$, is $(p, k)$-epi for each bounded open subset $\Omega$ containing $f^{-1}(p)$. We note that by virtue of our localization property for $(0, k)$-epi mappings it suffices to consider a sufficiently large open ball centered at the origin and containing $f^{-1}(0)$.

HOMOTOPY PROPERTY. If $f: E \rightarrow F$ is $\left(0, k_{1}\right)$-epi, $h:[0,1] \times E \rightarrow F$ is a $k_{2}$-set contraction such that $h(0, x)=0$, for all $x \in E, 0 \leqslant k_{2} \leqslant k_{1}<1$ and the set $S=\{x \in E: f(x)+h(x, t)=0$ for some $t \in(0,1]\}$ is bounded, then $f(\cdot)+$ $h(1, \cdot)$ is $\left(0, k_{1}-k_{2}\right)$-epi.

Proof. It is an easy consequence of our homotopy property for mappings on bounded sets.

THEOREM 3.1. Let $f: E \rightarrow F$ be $(0, k)$-epi with $k \in[0,1)$ and $Q \subset F$ be starshaped with respect to the origin. If $f^{-1}(Q)$ is bounded and $h: E \rightarrow F$ is a $k_{1}$-set contraction such that $0 \leqslant k_{1} \leqslant k<1$ and $\operatorname{Im} h \subset Q$, then the equation $f(x)=h(x)$ has a solution. In particular $\operatorname{Im} f \supset Q$.

Proof. Let $\Omega$ be a ball centered at the origin such that $\Omega \supset f^{-1}(Q)$. We consider the mapping $\left.f\right|_{\bar{\Omega}}: \bar{\Omega} \rightarrow F$. Then using a similar argument to that in Theorem 2.5 and the above homotopy property, the result follows.

Corollary 3.1. If $L: E \rightarrow F$ is a linear bounded mapping, then $L$ is $\left(0, t /\left\|L^{-1}\right\|\right)$-epi for each $t \in[0,1)$ if and only if $L$ is an isomorphism.

Proof. Let $L$ be an isomorphism. Then $L$ is continuous, injective and $\left\|L^{-1}\right\|$-proper. Hence $L$ is $\left(0, t / \| L^{-1}||\right)$-epi for each $t \in[0,1)$, by Corollary 2.1 . Conversely, if $L$ is $\left(0, t / \| L^{-1}||\right)$-epi, for all $t \in[0,1)$ then $L^{-1}(0)$ is bounded by the admissibility of $L$. Thus $L$ is one-to-one. To prove that $L$ is onto, let $p \in F$ and $Q=\{t p: 0 \leqslant t \leqslant 1\}$. Then $L^{-1}(Q)$ is clearly bounded. Hence by Theorem 3.1, $L(x)=p$ has a solution (taking $h$ to be the constant mapping $h(x)=p$ for all $x \in E$ ).

CoROllaRY 3.2. Let $f: E \rightarrow F$ be $(0, k)$-epi for some $k \in[0,1)$ and $\|f(x)\| \rightarrow \infty$ as $\|x\| \rightarrow \infty$. If $h: E \rightarrow F$ is a $k_{1}$-set contraction such that $0 \leqslant k_{1} \leqslant k<1$ and $h(E)$ is bounded, then the equation $f(x)=h(x)$ has a solution. In particular $f$ is onto. 
Proof. The condition $\|f(x)\| \rightarrow \infty$ as $\|x\| \rightarrow \infty$ implies that $f^{-1}(S)$ is bounded for every bounded subset $S$ of $F$. Since $\operatorname{Im} h=h(E)$ is bounded, we can take a ball $Q$ centered at the origin of $F$ sufficiently large that $Q \supset \operatorname{Im} h$. Hence the Corollary follows from Theorem 3.1.

REMARK 3.1. If $f$ and $h$ are as in Corollary 3.2, then $f+h$ is $\left(0, k-k_{1}\right)$-epi and, therefore, $f$ is $(p, k)$-epi for an $p \in F$.

THEOREM 3.2. Let $f: E \rightarrow F$ be 0 -admissible and $k_{1}$-proper on bounded closed sets and let $\left\{f_{n}: f_{n}: E \rightarrow F\right\}$ be a sequence of $(0, k)$-epi mappings such that $f_{n} \rightarrow f$ uniformly on closed bounded subsets of $E$. Further assume that the sets $f_{n}^{-1}(0)$ are uniformly bounded and $k k_{1}<1$. Then $f$ is $(0, k)$-epi.

Proof. This is a direct consequence of our Theorem 2.6.

\section{Applications}

EXAMPLE 1. In this example we try to find a solution $x \in C^{1}[0,1]$ of the following problem:

$$
A\left\{\begin{array}{l}
\dot{x}(t)=h(t, x(t), x(\phi(t)), \dot{x}(\phi(t))), \\
x(0)=d
\end{array}\right.
$$

where $\phi \in C[0,1]$ satisfies $0 \leqslant \phi(t) \leqslant t$ for all $t \in[0,1]$ and $h:[0,1] \times \mathbf{R}^{3} \rightarrow \mathbf{R}$ is continuous and satisfies

$$
|h(t, x, y, z)| \leqslant a+b|x|+c|y|+r|z|
$$

and

$$
|h(t, x, y, z)-h(t, x, y, u)| \leqslant r|z-y|
$$

for all $t \in[0,1]$ and $x, y, z, u \in \mathbf{R}, a, b, c$ and $r$ being nonnegative with $r<1$.

Furi, Martelli and Vignoli [1] considered the special case of this problem where $\dot{x}(t)=\mu(t) \dot{x}(\phi(t))+h(t, x(t), x(\phi(t)))$ where $\mu:[0,1] \rightarrow \mathbf{R}$ is continuous and satisfies $|\mu(t)| \leqslant t<1$ and $h:[0,1] \times \mathbf{R}^{2} \rightarrow \mathbf{R}$ is continuous and satisfies

$$
|h(t, x, y)| \leqslant a+b|x|+c|y|,
$$

where $a, b, c, r$ and $\phi$ are as above. Their method cannot be extended to treat the more general problem above. To this end we first prove the following results.

LEMMA 4.1. Let $a, b, c$ and $r$ be nonnegative numbers with $r<1$. Let $x$, $y \in C^{1}[0,1]$ and $\phi \in C[0,1]$ with $0 \leqslant \phi(t) \leqslant t$, for all $t \in[0,1]$. Further assume 
that for all $t \in[0,1]$ we have

$$
\begin{aligned}
& |\dot{x}(t)| \leqslant a+b|x(t)|+c|x(\phi(t))|+r|\dot{x}(\phi(t))|, \\
& \dot{y}(t)>a+b y(t)+c y(\phi(t))+r \dot{y}(\phi(t)),
\end{aligned}
$$

and

$$
|x(0)|<y(0)
$$

Then $|x(t)|<y(t)$, for all $t \in[0,1]$.

Proof. Since $\phi(0)=0$, we obtain by using (4.3), (4.4) and (4.5)

$$
\begin{aligned}
\dot{y}(0) & >[a+(b+c) y(0)] /(1-r) \\
& \geqslant[a+(b+c)|x(0)|] /(1-r) \geqslant|\dot{x}(0)| .
\end{aligned}
$$

Let $\tau=\sup \{t \in[0,1]:|x(s)|<y(s)$ and $|\dot{x}(s)|<\dot{y}(s), 0 \leqslant s<t\}$. By virtue of (4.5), (4.6) and continuity it follows that $0<\tau<1$.

Clearly

$$
|x(s)|<y(s) \text { and }|\dot{x}(s)|<\dot{y}(s) \text { for } s \in[0, \tau) .
$$

Suppose that $|x(\tau)|=y(\tau)$. Since $\dot{y}(s) \geqslant|\dot{x}(s)| \geqslant 0$ for $s \in[0, \tau]$ and $0 \leqslant \phi(s)$ $\leqslant s$, then $|x(\phi(s))| \leqslant y(\phi(s))$ and $\dot{y}(\phi(s)) \geqslant|\dot{x}(\phi(s))|$ for $s \in[0, \tau]$. Thus from (4.3) and (4.4) we obtain

$$
|\dot{x}(s)|<\dot{y}(s) \text { for } s \in[0, \tau) .
$$

This, together with (4.5), implies that $y(\tau)>|x(\tau)|$ which is a contradiction.

Suppose that $y(\tau)>|x(\tau)|$. Now since $|x(\phi(\tau))|<y(\phi(\tau))$ and $\dot{y}(\phi(\tau)) \geqslant$ $|\dot{x}(\phi(\tau))|$, it follows from (4.3) and (4.4) that $|\dot{x}(\tau)|<\dot{y}(\tau)$ which is again a contradiction. Thus the lemma is proved.

Corollary 4.1. Let $a, b, c$ and $r$ be nonnegative numbers with $r<1$. Let $x$, $y \in C^{1}[0,1]$ and $\phi \in C[0,1]$ with $0 \leqslant \phi(t) \leqslant t$, for all $t \in[0,1]$. Assume that $x$ satisfies (4.3) and $\dot{y}$ is nondecreasing. Further assume that

$$
\dot{y}(t)>a+b y(t)+c y(t)+r \dot{y}(t), \quad \text { for all } t \in[0,1]
$$

and $|x(0)|<y(0)$. Then $y(t)>x(t)$, for all $t \in[0,1]$.

Proof. Clearly $\dot{y}(0)>0$ and so $\dot{y}(t)>0$, for all $t \in[0,1]$. Thus $y(\phi(t)) \leqslant y(t)$. Again as $\dot{y}$ is nondecreasing, $\dot{y}(t) \geqslant \dot{y}(\phi(t))$. Hence (4.4) is satisfied and the result follows from the Lemma 4.1 .

COROllary 4.2. Let $a, b, c, r, x, y$ and $\phi$ be as in Lemma 4.1 and assume that (4.3) to (4.5) hold. Then $\dot{y}(t)>|\dot{x}(t)|$, for all $t \in[0,1]$. 
Proof. This is implicit in the proof of Lemma 4.1.

THEOREM 4.1. Let $h:[0,1] \times \mathbf{R}^{3} \rightarrow \mathbf{R}$ be continuous and satisfy (4.1) and (4.2). Then the mapping $H: C^{1}[0,1] \rightarrow C[0,1]$ defined by

$$
H(y)(t)=h(t, y(t), y(\phi(t)), \dot{y}(\phi(t)))
$$

is an r-set contraction.

Proof. Let $S \subset C^{1}[0,1]$ be a bounded set with $\alpha(S)=\lambda$. We show that $\alpha(H(S)) \leqslant r \lambda$ where $\alpha$ is the measure of noncompactness in the relevant spaces. Denote by $S^{\prime}$ the set $\left\{y^{\prime}: y \in S\right\}$ and identify $S$ and $S^{\prime}$ as subsets of $C$. Let $G$ : $C \times C \rightarrow C$ be defined by $G(u, v)(t)=h(t, u(t), u(\phi(t)), v(\phi(t)))$, for $t \in[0,1]$. as $H(S) \subseteq G\left(S, S^{\prime}\right)$ it suffices to show that $\alpha\left(G\left(S, S^{\prime}\right)\right) \leqslant r \alpha\left(S^{\prime}\right) \leqslant r \lambda$. Now the set $T=\left\{z: z=y(t)\right.$ or $z=y^{\prime}(t)$ for some $y \in S$ and $\left.t \in[0,1]\right\}$ is bounded. So given $\varepsilon>0$, by the uniform continuity of $h$ on $[0,1] \times T^{3}$, there is $\eta>0$ such that $|h(t, x, y, z)-h(t, p, q, z)|<\varepsilon$, whenever $|x-p|+|y-q|<2 \eta, t \in[0,1]$, and $x, y, z, p, q \in T$. As $S$ is a bounded equicontinuous subset of $C$ there is an $\eta$-net $u_{1}, \ldots, u_{m}$ for $S$ in $C$. Thus $G\left(S, S^{\prime}\right) \subseteq \bigcup_{i=1}^{m} B\left(G\left(u_{i}, S^{\prime}\right), \varepsilon\right)$ and by Lemma 1.2 and property (iv) of the measure of noncompactness

$$
\alpha\left(G\left(S, S^{\prime}\right)\right) \leqslant \max \left\{\alpha\left(G\left(u_{i}, S^{\prime}\right)\right)+2 \varepsilon: 1 \leqslant i \leqslant n\right\} .
$$

Thus it suffices to show that $\alpha\left(G\left(u_{i}, S^{\prime}\right)\right) \leqslant r \alpha\left(S^{\prime}\right)$ for $1 \leqslant i \leqslant m$. Let $V$ be an arbitrary bounded subset of $C$. We show that $\delta\left(G\left(u_{i}, V\right)\right) \leqslant r \delta(V)$, where $\delta$ denotes diameter in $C$. For $v_{1}, v_{2} \in V$,

$$
\left|G\left(u_{i}, v_{1}\right)(t)-G\left(u_{i}, v_{2}\right)(t)\right| \leqslant r\left|v_{1}(\phi(t))-v_{2}(\phi(t))\right|
$$

so $\left\|G\left(u_{i}, v_{i}\right)-G\left(u_{i}, v_{2}\right)\right\| \leqslant r\left\|v_{1}-v_{2}\right\|$ and the result follows.

Problem A. We now turn our attention to problem

$$
A\left\{\begin{array}{l}
\dot{x}(t)=h(t, x(t), x(\phi(t)), \dot{x}(\phi(t))), \\
x(0)=d
\end{array}\right.
$$

where $h$ satisfies (4.1) and (4.2) and $\phi \in C[0,1]$ with $0 \leqslant \phi(t) \leqslant t$ for $t \in[0,1]$.

Let $M: C^{1}[0,1] \rightarrow C[0,1] \times \mathbf{R}$ be defined by

$$
M(x)(t)=(\dot{x}(t)-h(t, x(t), x(\phi(t)), \dot{x}(\phi(t))), x(0)) .
$$

Then $M$ is $(0, k)$-epi for each $k \in[0,1-r)$. In particular the problem has a solution for each $d \in \mathbf{R}$.

Proof. We first note that $L: C^{1}[0,1] \rightarrow C[0,1] \times \mathbf{R}$ given by $(L x)(t)=$ $(\dot{x}(t), x(0))$ is an isomorphism with $\left\|L^{-1}\right\|=1$; here we use the norm in $C[0,1] \times \mathbf{R}$ defined by $\|(x, r)\|=\|x\|+|r|$ for $(x, r) \in C[0,1] \times \mathbf{R}$ where $\|x\|$ is the usual 
sup norm. Let $K: C^{1}[0,1] \rightarrow C[0,1] \times \mathbf{R}$ be defined by $K(x)(t)=$ $(h(t, x(t), x(\phi(t)), \dot{x}(\phi(t))), d)$. We claim that $S=\left\{x \in C^{1}[0,1]: L x=\lambda K(x)\right.$ for some $\lambda \in[0,1]\}$ is bounded. To this end let $L x=\lambda K(x)$ where $\lambda \in[0,1]$ and $x(0)=\lambda d$. By $(4.1)$

$$
|\dot{x}(t)| \leqslant a t+b|x(t)|+c|x(\phi(t))|+r|\dot{x}(\phi(t))| .
$$

Let $y(t)=a+(b+c) y(t)+r \dot{y}(t)+1$, that is, $\dot{y}(t)=[(1+a)+$ $(b+c) y(t)] /(1-r)$ and $y(0)=1+|d|$. By solving this equation we can easily see that $\dot{y}(t)$ is nondecreasing. Since $y(0)=1+|d|>|x(0)|=\lambda|d|$, by Corollaries 4.1 and 4.2 it follows that $\|x\| \leqslant\|y\|$ and hence $S$ is bounded.

From Theorem 4.1 it easily follows that $K$ is an $r$-set contraction and hence $H$ : $[0,1] \times C^{1}[0,1] \rightarrow C[0,1] \times \mathbf{R}$ defined by $H(\lambda, x)=-\lambda K(x)$ is also an $r$-set contraction. By our Corollary $3.1 L$ is $(0,1-\varepsilon)$-epi for each $\varepsilon \in[0,1)$. Hence by our homotopy property $L-K(\cdot)=M(\cdot)$ is $(0,1-\varepsilon-r)$-epi.

REMARK 4.1. It is not difficult to see that the above result can be strengthened by replacing (4.1) by

$$
|h(t, x, y, z)| \leqslant \psi(|x|)+\psi(|y|)+r|z|
$$

where $r \in[0,1)$ is a constant and $\psi:[0, \infty) \rightarrow(0, \infty)$ is a continuous strictly increasing function satisfying $\int^{\infty}(\psi(s))^{-1} d s=\infty$. All that is required is the corresponding modification of Lemma 4.1 and its corollaries and the observation that

$$
\dot{y}(t)=(2 \psi(|y(t)|)+1) /(1-r), \quad y(0)=1
$$

has a solution on $[0,1]$ with $\dot{y}(t)$ nondecreasing.

EXAMPLE 2 . Let $\Omega$ be a bounded $C^{1,1}$ domain in $\mathbf{R}^{n}$, let $L^{p}(\Omega)$ be the spaces of Lebesgue measurable functions with the usual norms, for $1 \leqslant p \leqslant \infty$, and let $W_{0}^{p, q}(\Omega)$ and $W^{p, q}(\Omega)$ be the usual Sobolev spaces for $p, q=1,2, \ldots$ Let $a_{i j} \in C(\bar{\Omega}), b_{i}, c \in L^{\infty}(\Omega)$ for $i, j=1,2, \ldots, n$, and let

$$
a_{i j}(x) \xi_{i} \xi_{j} \geqslant \lambda_{0}|\xi|^{2}
$$

for all $\xi \in \mathbf{R}^{n}, x \in \Omega$ and, some constant $\lambda_{0}>0$ and $c \leqslant 0$. Let $h: \Omega \times \mathbf{R} \times \mathbf{R}^{n}$ $\times \mathbf{R}^{n^{2}}$ satisfy

$$
|h(x, u, p, s)| \leqslant k\{|u|+|p|+|s|\}+g(x)
$$

for some constant $k>0$ and $g \in L^{2}(\Omega)$ and

$$
|h(x, u, p, s)-h(x, u, p, t)| \leqslant k|s-t|,
$$

for all $(x, u, p) \in \Omega \times \mathbf{R} \times \mathbf{R}^{n}$ and for all $s, t \in \mathbf{R}^{n^{2}}$. Moreover let $h$ satisfy the Carathéodory conditions: that is, let $h(x, \cdot, \cdot, \cdot): \mathbf{R} \times \mathbf{R}^{n} \times \mathbf{R}^{n^{2}}$ be continuous a.e. $x \in \Omega$ and let $h(\cdot, u, p, s): \Omega \rightarrow \mathbf{R}$ be measurable for all $(u, p, s) \in \mathbf{R} \times \mathbf{R}^{n}$ $\times \mathbf{R}^{n^{2}}$. 
Let $B=W_{0}^{1,2}(\Omega) \cap W^{2,2}(\Omega)$ with the $W^{2,2}$ norm and define $L: B \rightarrow L^{2}(\Omega)$ by $L u=a_{i j} D_{i j} u+b_{i} D_{i} u+c u$. We look for a solution $u \in B$ of the Dirichlet problem

$$
B\left\{L u=\varepsilon h\left(x, u, D u, D^{2} u\right) \text { in } \Omega,\right.
$$

where $\varepsilon>0$ is sufficiently small.

Define the Nemitsky operator $M: L^{2}(\Omega) \times\left(L^{2}(\Omega)\right)^{n} \times\left(L^{2}(\Omega)\right)^{n^{2}} \rightarrow L^{2}(\Omega)$ by $M(u, p, s)(x)=h(x, u(x), p(x), s(x))$. From the growth on $h, M$ is continuous (see [10], page 162). By the Kondrachov compactness theorem (see [2], page 167) the mapping $K: B \rightarrow L^{2}(\Omega) \times\left(L^{2}(\Omega)\right)^{n}$ given by $K(u)=(u, D u)$ is completely continuous. We show that the mapping $H: B \rightarrow L^{2}(\Omega)$ given by $H(u)=$ $M\left(K(u), D^{2} u\right)$ is a $2 k$-set contraction. As $M, K$ and $D^{2}$ are continuous, $H$ is continuous. We show that for any bounded set $S \subseteq B, H(S) \subseteq H_{1}(S)+H_{2}(S)$ where $\overline{H_{2}(S)}$ is compact and $\alpha\left(H_{1}(S)\right) \leqslant 2 k \alpha(S)$; here $\delta$ and $\alpha$ represent the diameter and the measure of noncompactness, respectively, in the appropriate spaces and for $G: B \rightarrow L^{2}(\Omega), G(S)=\{G(u): u \in S\}$. Fix $w \in S$ and define $H_{1}, H_{2}: B \rightarrow L^{2}(\Omega)$ by $H_{2}(u)=M\left(K(u), D^{2} w\right)$ and $H_{1}(u)=H(u)-H_{2}(u)$ for all $u \in B$. As $\left|D^{2} w\right| \in L^{2}(\Omega)$ it follows that $M\left(\cdot, \cdot, D^{2} w\right): L^{2}(\Omega) \times\left(L^{2}(\Omega)\right)^{n} \rightarrow$ $L^{2}(\Omega)$ is continuous and hence $H_{2}$ is completely continuous. We show that $\delta\left(H_{1}(S)\right) \leqslant 2 k \delta(S)$ and then $\alpha\left(H_{1}(S)\right) \leqslant 2 k \alpha(S)$ as $S$ is an arbitrary bounded set. By the Lipschitz condition on $h,\left|h\left(x, u, D u, D^{2} u\right)-h\left(x, u, D u, D^{2} w\right)\right| \leqslant$ $k\left|D^{2} u-D^{2} w\right|$ so that $\left\|H_{1}(u)\right\| \leqslant k\|u\|$ and hence $\delta\left(H_{1}(S)\right) \leqslant 2 k \delta(S)$. Now $\alpha(H(S)) \leqslant \alpha\left(H_{1}(S)\right)+\alpha\left(H_{2}(S)\right) \leqslant \alpha\left(H_{1}(S)\right) \leqslant 2 k \alpha(S)$, as required.

By Theorem 9.15 and Corollary 9.17 of Gilbarg and Trudinger [2] $L: B \rightarrow$ $L^{2}(\Omega)$ is an isomorphism. Choose $\varepsilon_{0}>0$ such that $2 k \varepsilon_{0} l^{-1}<1$ where $l^{-1}=\left\|L^{-1}\right\|$. Let $S=\left\{(\lambda, u) \in[-1,1] \times B: L u=\lambda \varepsilon_{0} H(u)\right\}$. We show that $S$ is bounded. Let $(\lambda, u) \in S$ then $\left\|\lambda \varepsilon_{0} H(u)\right\| \leqslant \varepsilon_{0}\|g\|+k \varepsilon_{0}\|u\|$ thus $\|u\| \leqslant l^{-1} \varepsilon_{0}\|g\|+k \varepsilon_{0} l^{-1}\|u\|$ and $\|u\| \leqslant 2 l^{-1} \varepsilon_{0}\|g\|$. As $\varepsilon H$ is a $2 \varepsilon_{0} k$-set contraction for $|\varepsilon| \leqslant \varepsilon_{0}, 2 \varepsilon_{0} k l^{-1}<1$, and $L$ is an isomorphism with $\left\|L^{-1}\right\|=l^{-1}$, then $L-\varepsilon H$ is $(0, \bar{k})$-epi for each $\bar{k} \in\left[0, l-2 \varepsilon_{0} k\right)$, by Corollary 3.1 and the homotopy property of Section 3 . Now problem B is equivalent to finding a solution $u \in B$ of $L u=\varepsilon H(u)$ so problem B has a solution for each $\varepsilon$ with $|\varepsilon| \leqslant \varepsilon_{0}$.

Problem $\mathrm{C}$ of the next example is well known. There are several different existence proofs in the literature. We give an existence proof based on the previous sections of this paper.

EXAMPLE 3. Let $X$ be a Banach space with norm denoted by $\mid \cdot$. Let $C([0,1], X)$ be the space of continuous functions $y:[0,1] \rightarrow X$ with norm defined by $\|y\|=\sup \left\{e^{-t t}|y(t)|: 0 \leqslant t \leqslant 1\right\}$, where $l>1$ is a constant given below. Let $C^{1}([0,1], X)$ be the space of continuously differentiable functions $y$ in $C([0,1], X)$ with norm $\|y\|=\max \left\{\|y\|, \frac{1}{t}\left\|y^{\prime}\right\|\right\}$. Clearly a bounded subset $S \subseteq C^{1}$ is a 
bounded equicontinuous subset of $C$. Let $h$ : $[0,1] \times X \rightarrow X$ be uniformly continuous and assume there is a constant $k>0$ such that $|h(t, y)| \leqslant k$, for all $(t, y) \in[0,1] \times X$, and $\alpha(h(t, S)) \leqslant k \alpha(S)$, for all bounded subsets $S \subseteq X$. Then there is a solution $y \in C^{1}$ of the problem

$$
C\left\{\begin{array}{l}
y^{\prime}=h(t, y), \quad \text { for all } t \in[0,1], \\
y(0)=y_{0} .
\end{array}\right.
$$

It suffices to consider the case $y_{0}=0$. Let $C_{0}^{1}([0,1], X)=\left\{y: y \in C^{1}, y(0)=0\right\}$. Clearly $C_{0}^{1}$ is a closed subset of $C^{1}$.

Define $L: \quad C_{0}^{1} \rightarrow C$ by $(L y)(t)=y^{\prime}(t)$ and $H: C_{0}^{1} \rightarrow C$ by $H(y)(t)=$ $h(t, y(t))$. If $S \subseteq C_{0}^{1}$ is a bounded set then for an arbitrary bounded subset $D \subseteq C$ and $t \in[0,1]$ let $D(t)=\{y(t): y \in D\}$. If $S \subseteq C_{0}^{1}$ is a bounded set then $H(S)=\{H(y): y \in S\}$ is a bounded equicontinuous subset of $C$ so by variants of Lemma 2.3.1 and Theorem 2.3.1 of Martin [8]

$$
\begin{aligned}
\alpha(H(S)) & =\sup \left\{e^{-l t} \alpha(H(S)(t)): 0 \leqslant t \leqslant 1\right\} \\
& \leqslant \sup \left\{e^{-l t} k \alpha(S(t)): 0 \leqslant t \leqslant 1\right\} \\
& =k \alpha(S) .
\end{aligned}
$$

Clearly $H$ is continuous and it is not difficult to show that $L$ is an isomorphism with $\left\|L^{-1}\right\| \leqslant l^{-1}$. Choose $l>k$. Now the problem $\mathrm{C}$ is equivalent to solving $L y=H(y)$. Let $(\lambda, y) \in S=\left\{(\lambda, y) \in[0,1] \times C_{0}^{1}: L y=\lambda H(y)\right\}$, then for all $t \in[0,1], y^{\prime}(t)=\lambda h(t, y(t))$ and $y(0)=0$. Thus $|\dot{y}(t)| \leqslant k$ and hence $|y(t)| \leqslant$ $k$, for all $t \in[0,1]$. Hence $\|y\| \| \leqslant$ and $S$ is bounded.

The result now follows by an argument similar to that in Example 2. In particular $L-H$ is $(0, \bar{k})$-epi for $\bar{k} \in\left[0,\left\|L^{-1}\right\|^{-1}-k\right)$ and hence problem $C$ has a solution.

ExAmple 4. Let $\phi \in C^{2}(\mathbf{R})$ satisfy $\phi^{\prime}(x)<0$ and $\phi^{\prime \prime}(x)>0$, for all $x \in \mathbf{R}$ and set $\Omega=\left\{(x, y) \in \mathbf{R}^{2}: y>\phi(x)\right\}$. Let $h: \bar{\Omega} \times \mathbf{R}^{3} \rightarrow \mathbf{R}$ be continuous. Let $\bar{y}=$ $\phi(x)$ and $\bar{x}=\phi^{-1}(y)$ and for $u: \bar{\Omega} \rightarrow \mathbf{R}$ let

$$
\|u\|=\sup \left\{e^{-l[x-\bar{x}+y-\bar{y}]}|u(x, y)|:(x, y) \in \bar{\Omega}\right\},
$$

where $l>1$ is chosen later. Let $C(\bar{\Omega})=\{u: \bar{\Omega} \rightarrow \mathbf{R}, u$ is continuous, $\|u\|<\infty\}$.

Consider the Cauchy problem

$$
D \begin{cases}u_{x y}(x, y)=h\left(x, y, u, u_{x}, u_{y}\right) & \text { in } \Omega, \\ u=u_{x}=u_{y}=0 & \text { on } \partial \Omega,\end{cases}
$$

where a solution $u$ is a function satisfying $u, u_{x}, u_{y}, u_{x y}=u_{y x} \in C(\bar{\Omega})$ and $D$ pointwise.

We assume that $h$ satisfies

$$
|h(x, y, u, p, q)-h(x, y, u, s, t)| \leqslant k\{|p-s|+|q-t|\},
$$


and

$$
|h(x, y, u, p, q)| \leqslant k\{|u|+|p|+|q|\}+g(x, y)
$$

for all $(x, y) \in \Omega$ and for all $p, q, r, s \in \mathbf{R}$, where $g \in C(\bar{\Omega})$. We show that problem $D$ has a solution. By translating axes we may assume that $(0,0) \in \partial \Omega$. Let $\Omega_{n}=\{(x, y) \in \Omega:|x|,|y|<n\}$, for all $n=1,2, \ldots$, and let $C\left(\bar{\Omega}_{n}\right)$ and $\|u\|$ for $u \in C\left(\bar{\Omega}_{n}\right)$ have the obvious interpretations. We find a solution $u_{n}$ of problem

$$
D_{n} \begin{cases}u_{x y}(x, y)=h\left(x, y, u, u_{x}, u_{y}\right) & \text { in } \Omega_{n}, \\ u=u_{x}=u_{y}=0 & \text { in } \partial \Omega \cap \bar{\Omega}_{n},\end{cases}
$$

and show problem $\mathrm{D}$ has a solution by a standard diagonalization argument. Let

$$
B_{n}=\left\{u: u, u_{x}, u_{y} \text {, and } u_{x y}=u_{y x} \in C\left(\bar{\Omega}_{n}\right), u=u_{x}=u_{y}=0 \text { on } \partial \Omega \cap \bar{\Omega}_{n}\right\}
$$

and define a norm on $B_{n}$ by $\|u\|=\|u\|+\left\|u_{x}\right\|+\left\|u_{y}\right\|+l^{-1}\left\|u_{x y}\right\|$. Thus $B_{n}$ is a Banach space. Let $L, N: B_{n} \rightarrow C\left(\bar{\Omega}_{n}\right)$ be given by $(L u)(x, y)=u_{x y}(x, y)$ and $H(u)(x, y)=h\left(x, y, u, u_{x}, u_{y}\right)$, for all $u \in B_{n}$ and for all $(x, y) \in \bar{\Omega}_{n}$. It is not difficult to show that problem $D_{n}$ is equivalent to solving $L u=H(u)$. Now $L$ is an isomorphism with $\left\|L^{-1}\right\| \leqslant l^{-1}$. Choose $l>2 k$. Now $H$ is a $k$-set contraction. This follows by a similar argument to that in Example 1 since a bounded subset of $B_{n}$ has compact closure in $C\left(\bar{\Omega}_{n}\right)$. Let $S_{n}=\left\{(\lambda, u) \in[0,1] \times B_{n}: L u=\lambda H(u)\right\}$ then $S_{n}$ is bounded independently of $n$. To see this $(\lambda, u) \in S_{n}$ then $\|\lambda H(u)\| \leqslant$ $k\|u\|\|+\| g \|$. Since $\left\|L^{-1}\right\| \leqslant(2 k)^{-1}$ then $\|u\|\left\|\leqslant k^{-1}\right\| g \| \leqslant C$, a constant as $g \in C(\bar{\Omega})$. By an argument similar to that in Example 2, there is a solution $u_{n} \in B_{n}$ of $L u=H(u)$. Now let $T_{n}=\left\{u \in B_{n}: L u=H(u)\right\}=(L-H)^{-1}(\{0\})$. By Theorem 1.5, $\alpha\left(T_{n}\right)=0$ since $k_{1} k=\left\|L^{-1}\right\| k<1$. Clearly $\left.u_{m}\right|_{\bar{\Omega}_{n}} \in T_{n}$, for all $m \geqslant n$. Choose a subsequence of $u_{m}$ denoted by $u_{m, 1}$, after relabelling, such that $u_{m, 1} \mid \tilde{s}_{1}$ converges in $B_{1}$. Proceeding inductively we may choose a subsequence of $u_{m, n}$ denoted by $u_{m, n+1}$, after relabelling, such that $u_{m, n+1} \mid \bar{\Omega}_{n}$ converges in $B_{n+1}$. Thus $u=\lim _{n \rightarrow \infty} u_{n, n}$ with the limit appropriately interpreted is a solution of problem $\mathrm{D}$, as required.

Remark 4.2. Problem D is similar to one studied by Hartman and Wintner [5]; however they establish existence in a bounded domain by producing a convergent sequence of approximate solutions. Thus, our existence result in a bounded domain is cleaner. It would be interesting to know if the existence of a solution in the unbounded domain could be established directly without finding solutions in an expanding sequence of bounded domains. In the case $h$ is Lipschitz with respect to $u$ as well as $u_{x}$ and $u_{y}$ the existence of a solution on all of $\Omega$ follows directly from the contraction mapping principle, as is well known. 


\section{Acknowledgement}

We thank the referee for drawing our attention to the work of Fitzpatrick and Petryshyn (Some applications of $A$-proper mappings, Nonlinear Analysis, Theory, Methods \& Applications 3 (1979), 525-532) in which they consider a problem of the form

$$
L u=g\left(x, u, \nabla u, D^{2} u\right)+h(x, u, \nabla u, L u), \text { for all } x \in \Omega,
$$

where $L$ is a linear second order elliptic partial differential operator from $W^{2,2}(\Omega) \cap \dot{W}^{1,2}(\Omega)$ to $L^{2}(\Omega)$. Although there is some formal similarity with our problem B of Example 2, their problem is somewhat different from, and their results do not apply to, our problem $B$.

\section{References}

[1] M. Furi, M. Martelli and A. Vignoli, 'On the solvability of nonlinear operator equations in normed spaces', Ann. Mat. Pura Appl. 124 (1980), 321-343.

[2] D. Gilbarg and N. S. Trudinger, Elliptic partial differential equations of second order (SpringerVerlag, New York, 1983).

[3] I. C. Gokhberg, L. S. Goldstein and A. S. Markus, 'Investigation of some properties of bounded linear operators in connection with $q$-norms', Uchen. Zap. Kishinev. Gos. Univ. 29 (1957), 29-36.

[4] A. Granas, 'The theory of compact vector fields and some applications to the theory of functional spaces', Rozprawy Matematyezne, Warszawa, 30 (1962).

[5] P. Hartman and A. Wintner, 'On hyperbolic partial differential equations,' Amer. J. Math. 74 (1952), 834-864.

[6] K. Kuratowski, 'Sur les espaces comptets', Fund. Math. 15 (1930), pp. 301-319.

[7] N. G. Lloyd, Degree theory (Cambridge University Press, London, 1978).

[8] Robert H. Martin, Jr., Nonlinear operators and differential equations in Banach spaces (John Wiley and Sons, New York, 1976).

[9] R. D. Nussbaum, 'The fixed point index for local condensing maps', Ann. Mat. Pura Appl. 124 (1971), 217-258.

[10] M. M. Vainberg, Variational methods for the study of nonlinear operators (Holden-Day Inc., London, 1964).

\section{Department of Mathematics}

University of Queensland

St. Lucia, Queensland

Australia 4067 